\title{
Using Drones for Virtual Tourism
}

\author{
David Mirk and Helmut Hlavacs \\ University of Vienna, Research Group Entertainment Computing, Vienna, Austria \\ david@mirk.at, helmut.hlavacs@univie.ac.at
}

\begin{abstract}
In this paper we evaluate the usability of flying drones in tourism. A live video feed is streamed live from a drone to a tourist at some distance, wearing only a VR visor, and steering the drone with head movements.
\end{abstract}

\section{$1 \quad$ Introduction}

Virtual reality offers tourism many useful possibilities to create or extend virtual experiences that tourists may accept and use as partial alternatives for real visitation $[4,5]$. Especially in some areas of tourism, e.g. marketing, entertainment or education virtual reality will become more and more valuable. The vision of this work is to allow users of a client software application to receive real-time video images of different places of the world and watch them locally. As a special feature, and unlike to fixed stationary webcams, the user should be as free as possible to define where the "Virtual Eye" resides and what it is looking at.

\section{The Virtual Tourist Project}

Our approach is to use a cheap off-the-shelf drone (a Parrot ARDrone 2.0 [6] [8]) as a flying camera, and be used in a live scenario by tourists to roam around remote areas and ideally be immersed in the application such that the tourist has the impression of really being there.

To fulfill the task as complete as possible, we decided to choose a development pattern which divides the control application to be developed into three main components. Firstly, the server application, which is responsible for the direct control of the ARDrone, transmitting the calculated navigation vectors to the ARDrone. The second main component defines the graphical user client (client application), which processes and transmits the direct input to the ground station via TCP. Finally, the existing SDK for this purpose must be modified and extended in order to allow the user to use a wide a range of different control devices (e.g. "Oculus Rift").

The ARDrone 2.0 is very susceptible to outer influences. Moderate wind gusts deviate the drones very quickly from their original course which leads to a behavior where the electronics of the quadrocopter try to counteract this divergence with abrupt control maneuvers. This makes it difficult to provide a stable (vibration-free) video sequence to the user $[1,2,3]$. Furthermore, the rigid mounting of the video cameras 
leads to an undesirable side effect: basics quadrocopters control their flight direction by changing the speeds of their propellers and thus changes their attitude. At an acceleration to the front the drone tilts with the front camera toward the ground, on a flight back into sequence then turned toward the sky. To avoid this effect a modification with a steerable front camera on a ball joint would be necessary.

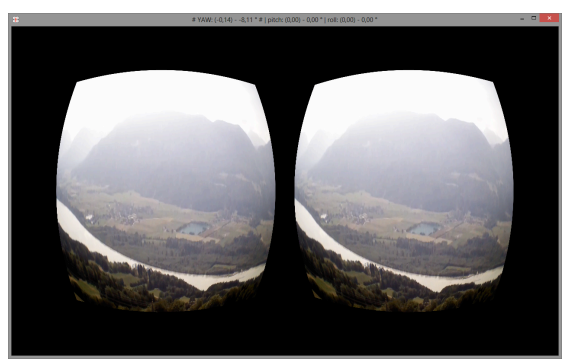

Fig. 1. View through Oculus Rift (Carinthia/Austria)

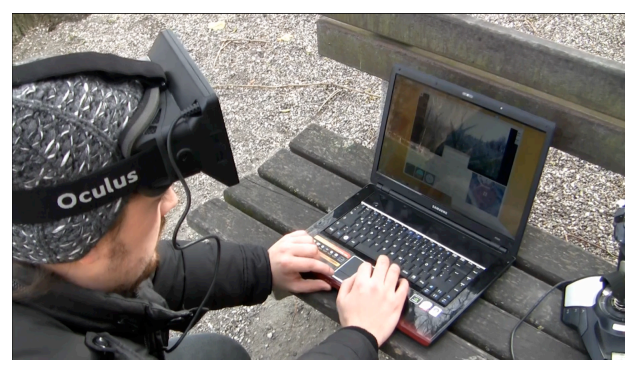

Fig. 2. Outdoor

A practical test arrangement showed that it is not possible to give the user complete freedom of the control over his permit virtual eyes. For this reason, a virtual route in the form of GPS waypoints must be predefined, on which the ARDrone travels within certain tolerances. As a result the user directly controls only the flight speed of the virtual tour, not the drone navigation directions. However, this allows a completely free focus and control of the front camera orientation.

\subsection{Navigation}

Due to the small narrow field of view of 91 degree and the rigidly mounted front camera a user-controlled direct navigation of the ARDrone would only be possible after several exercise passages. The specification of a flight course, which on the one hand is free of any obstacles (thus the risk of collisions decreases), and on the other hand provides optimized designed sightseeing courses (i.e., they lie within the flight range of the ARDrone and show the most important sights), allows the user to enjoy a satisfactory experience without the need of having knowledge of the actual flight behavior and functional ways of the quadrocopter.

\subsection{Virtual Reality}

The ARDrone 2.0 and the provided SDK already provides numerous possibilities for real-time symbol or character recognition to developers. Necessary calculations are made directly on the ARDrone hardware/firmware and the results are processed in a client application. The Oculus Rift is a virtual reality headset, originally designed for immersive gaming. To create a strong sense of immersion it is designed to almost fill 
the wearer's entire field of view and to completely block out the real world. The pincushioned image for each eye $(640 \times 800$ resolution $)$ is corrected by lenses in the headset. The used combination of 3-axis gyros, accelerometers, and magnetometers make it capable of absolute (relative to earth) head orientation tracking without drift $[8,9,10]$.

\section{Conclusion and Future Work}

The aim of this project was to develop a system that allows a user from anywhere on the world to control a ARDrone 2.0 via the Internet where the direct control of the direction of the quadrocopter is exclusively managed by the server application and only the alignment of the front camera is assumed by the user. However, the difficulties and problems that occurred while developing and testing this solution with the consumer grade Parrot ARDrone 2.0, led us to the conclusion that for a practicable use of the Virtual Tourist in tourism, new quadrocopter model has to be chosen, which fulfills more of the given requirements.

\section{References}

1. Balas, C.: Modelling and linear control of a quadrotor. MSc thesis, School of Engineering, Craneld University, 2007.

2. Brooks, A.C.: Real-time digital image stabilization. Image Processing Computer Project Final Paper, Northwestern University, USA (2003).

3. Czyba, R.: Attitude stabilization of an indoor quadrotor. European Micro Aerial Vehicle Conference and Competition (2009).

4. Guttentag, D.A.: Virtual reality: Applications and implications for tourism. Tourism Management 31(5), 637-651 (2010).

5. Letellier, R.: Virtual reality - a new tool for sustainable tourism and cultural heritage sites management. Heritage Group (1999)

6. Parrot AR.drone open api platform, https://projects.ardrone.org/

7. Sarumi, O. A., Balogun, V.F., Thompson, A.F.: A $3 \mathrm{~d}$ geo spatial virtual reality system for virtual reality. Pacific Journal of Science 11(29, 601-609 (2010).

8. Mondragon, I., Olivares, M., Campoy, P., Martinez, C., Mejias, I.: Unmanned aerial vehicles uavs attitude, height, motion estimation and control using visual systems. Auton Robot 29(1), 17-34 (2010).

9. Tico, M., Vehvilainen, M.: Robust method of digital image stabilization. In: Proc. of 3rd International Sumposium on Communications, Control and Signal Processing (ISCCSP), IEEE (2008)

10. Silven, O., Niskanen, M., Tico, M.: Video stabilization performance assessment. In: Proc. of IEEE International Conference on Multimedia and Expo (ICME), IEEE (2006) 